\title{
Reversal of chemosensitivity and induction of cell malignancy of a non-malignant prostate cancer cell line upon extracellular vesicle exposure
}

\author{
Kiriaki Panagopoulos ${ }^{1 \dagger}$, Sam Cross-Knorr ${ }^{2 \dagger}$, Christen Dillard ${ }^{2}$, Dionysios Pantazatos ${ }^{1,2}$, Michael Del Tatto ${ }^{2}$, \\ David Mills's, Lisa Goldstein ${ }^{3}$, Joseph Renzulli ${ }^{4}$, Peter Quesenberry ${ }^{2}$ and Devasis Chatterjee ${ }^{2^{*}}$
}

\begin{abstract}
Background: Extracellular vesicle (EV) trafficking is a fundamental cellular process that occurs in cells and is required for different aspects of pathophysiology. EV trafficking leads to changes in cellular function including apoptosis, angiogenesis and proliferation required for increased tumor formation.

Results: We report several phenotypic changes mediated by EVs isolated from non-malignant and malignant prostate cells as well as patient biopsied prostate tumor samples. EVs can reverse the resistance of prostate cancer cells to camptothecin EVs isolated from non-malignant PrECs (Prostate Epithelial Cells) can reverse soft agar colony formation of malignant DU145 cells, with the reciprocal effect observed. Isolation of EVs from 2 Gleason grade 8 prostate cancer patients significantly induced soft agar colony formation of non-malignant PrECs. We have identified proteins via antibody and Mass spectrometry analysis that may be responsible for the phenotypic changes. Mass spectrometry analysis of protein lysates using ProteolQ revealed protein candidates associated with gene ontology annotations that may be responsible for this phenotypic change. Ingenuity Pathway Analysis was used to identify statistically relevant canonical pathways and functions associated the protein IDs and expression values obtained using ProteolQ. Western blot analysis confirmed the increase of 14-3-3 zeta, pRKIP and prohibitin protein levels in PrEC cells co-cultured with patient EVs. 14-3-3 proteins were also found as common proteins of 3 other Gleason grade 8 patients.
\end{abstract}

Conclusion: Our study provides a rational basis to further investigate putative proteins, such as 14-3-3 and prohibitin and genetic factors that may be responsible for phenotypic changes that are associated with prostate cancer progression.

Keywords: Extracellular vesicles, Liquid chromatography-tandem mass spectrometry, Prostate cancer, Proteomics, Gene ontology

\section{Introduction}

Prostate cancer is the most frequently diagnosed malignancy and ranks second amongst all cancers in men with an estimated 218,890 men in the United States every year and 27,350 deaths per year (American Cancer Society 2007). Patients enjoy a 5 -year survival rate approaching nearly $100 \%$. However, as evidence of the slow but steady nature of this disease, $30-40 \%$ of patients will

\footnotetext{
* Correspondence: devasis_chatterjee@brown.edu

${ }^{\dagger}$ Equal contributors

${ }^{2}$ Division of Hematology/Oncology and Department of Medicine, Rhode Island Hospital, Providence, RI, USA

Full list of author information is available at the end of the article
}

experience a PSA recurrence within 10 years following definitive surgery or radiation therapy [1]. Patients with high risk or advanced disease or staging, or who have suffered a recurrence, historically receive treatment with androgen ablation therapy. Sometimes these failures are supplemented with salvage radiation therapy and possibly receive chemotherapy [2-5]. The progression of disease is inevitable and as a result, the median survival in advanced disease is only 18 to 20 months with an overall survival of 24 to 36 months. Therefore, there is an urgent need to identify novel genes or proteins that may be useful in selecting patients for mechanism-based therapeutics to improve clinical outcome.

\section{() Biomed Central}

(c) 2013 Panagopoulos et al.; licensee BioMed Central Ltd. This is an open access article distributed under the terms of the Creative Commons Attribution License (http://creativecommons.org/licenses/by/2.0), which permits unrestricted use, distribution, and reproduction in any medium, provided the original work is properly cited. 
Recent attention has been focused on the task of identifying soluble factors secreted by tumor cells and characterizing their paracrine activities [6-10]. In addition to soluble paracrine factors, many tumor cells also release extracellular vesicles (EVs), microvesicles (MVs) or exosomes. These vesicles are distinguished by their size (30-1000 nm) and morphology and are secreted by a variety of cell types under physiological and pathological conditions; specifically they are secreted when a multivesicular endosome fuses with the plasma membrane [11,12]. According to previous studies, EVs can contain bioactive molecules, nucleic acids, and/or proteins [13]. Interestingly, the abundance of EVs released generally correlates positively with advanced grade and stage of cancer progression [14]. Activated cells of various types are known to produce and shed membrane EVs into their surroundings. However, the mechanism triggering EV generation by cancer cells is unknown. There is mounting evidence that vesicle trafficking is a highly important process in tumorigenesis. Further evaluation of vesicle trafficking may reveal a number of targets and strategies that may be important for cancer therapeutics.

Previously, it has been shown that various aspects of cellular phenotype can be transferred from one cell type to another via EVs. Accordingly there has been a strong focus on the use of EVs as a vaccine in cancer [15]. This includes the transfer of cell surface molecules of mRNA and of apoptotic bodies. One study investigated the secretion of EVs from the human prostate cancer cell lines, DU145 and LNCaP, and showed an association with a region of frequent chromosomal deletion in metastatic disease [16]. This work suggested that EVs shed from prostate cancer cells could alter the tumor microenvironment in a manner that may promote disease progression. A recent publication has demonstrated that proteins found in PC-3 cell released EVs that are mainly involved in transport, cell organization and biogenesis, metabolic process, response to stimulus, and regulation of biological processes [17].

In this study we confirmed that EVs could be utilized as a potential mechanism of suppressing growth and reversing the cancerous phenotype. We demonstrate that we can reverse the resistance of prostate cancer cells to CPT via EVs as measured by apoptosis, cytotoxicity, and growth in soft agar. In addition, growth in soft agar, a hallmark of malignant cells, can be inhibited when DU145 cells are co-cultured with EVs isolated from nonmalignant human prostate epithelial (PrEC) cells with the reciprocal result occurring with PrEC cells cocultured with DU145 EVs. Phosphoproteomic analysis revealed the transfer of numerous proteins in our coculture model. Two proteins of significance, Suppressor of cytokine signaling 3 (SOC3) and Signal transducer and activator of transcription 3 (STAT3) were acquired or inhibited by co-culture of PrEC EVs with DU145 cells. Similarly, we found the increase in 14-3-3 zeta/delta phosphorylated Raf kinase inhibitor protein (pRKIP) and prohibitin from EVs isolated from 2 patient samples and co-cultured with PrEC. 14-3-3 zeta/delta/eta was also found as a common protein from 3 other Gleason grade 8 patients. The link between these proteins with cell survival, apoptosis induction, and tumor promotion provide a rational basis for therapeutic intervention. Therefore our study provides the basis for examining proteins released by EVs that are associated with disease progression and phenotype switching.

\section{Materials and methods}

Materials All reagents and chemicals were purchased from Sigma Chemical Co. (St. Louis, MO) unless otherwise noted. ST2461, a CPT analog, was provided by Sigma Tau (Rome, Italy). Protein quantification reagents were obtained from Bio-Rad Laboratories, Inc. (Hercules, CA). Enhanced chemiluminescence reagents and secondary mouse and rabbit horseradish peroxidase-conjugated antibodies for Western blot analysis were ordered from GE Healthcare (Arlington Heights, IL). The antibody to RKIP was purchased from Millipore (Hopkington, MA); the actin-HRP, pRKIP, SOCS3, prohibitin, 14-3-3 zeta and STAT3 antibodies were purchased from Santa Cruz Biotechnology (Santa Cruz, CA); and the PARP antibody was purchased from Invitrogen (Carlsbad, CA).

\section{Cells}

The human prostate carcinoma cell line DU145 was purchased from ATCC (Rockville, MD). The RC1 cell line, which was derived from DU145 cells, has been described [18]. The cell lines in our lab were used between passage numbers 10-20. The cells were grown in RPMI 1640 medium and supplemented with $10 \%$ fetal calf serum, glutamine, non-essential amino acids, 100 units/ml penicillin and 50 units $/ \mathrm{ml}$ streptomycin and cultured in a humidified incubator at $37^{\circ} \mathrm{C}$ containing $5 \% \mathrm{CO}$. The PrECs utilized in this study were obtained from Dr. William Hahn at Dana Farber Cancer Institute, Boston, MA [19] and were gown in PrEBM media supplemented with PrEGM SingleQuots (Lonza, Walkersville, MD). PrECs were used in between passage 2 and 10 .

\section{Western blot analysis}

Total cell extracts were prepared as previously described [18]. Protein concentrations of lysates were determined using the Bradford assay kit (BioRad). Proteins were separated by SDS-PAGE and electrophoretically transferred from the gel to nitrocellulose membranes (GE Healthcare). Proteins recognized by the antibodies were detected by enhanced chemilluminescence reagents (GE Healthcare). 


\section{Tissue collection}

Consent was obtained according to the Rhode Island Hospital's Committee on Protection of Human Subjects (Institutional Review Board) for each of the patients involved. In collaboration with our colleagues, we obtained fresh prostate tissue specimens following robotic assisted laparoscopic prostatectomy (Da Vinci Robotic Surgical System at The Miriam Hospital). In accordance with the requirements mandated by the Department of Health the active robotic surgeons have maintained a detailed database with preoperative, intraoperative and postoperative parameters recorded for each case. We isolated EVs from two patients; Patient 18: Gleason $4+4=8$ with tertiary 5 pattern (high risk) prostate cancer with positive margin focally and seminal vesicle invasion (pathology stage T3b). Patient 19: Gleason $4+4=8$ with tertiary 5 pattern (high risk) prostate cancer with negative margins (pathology stage T3a). Patients 13, 14, and 16 were also Gleason grade 8 .

\section{Extracellular vesicle isolation}

Extracellular vesicle isolation was conducted for five different patient cancer tissue samples with Institutional Review Board approval at Rhode Island Hospital. Tumor samples were weighed and minced with a sterile scalpel into 1-2 $\mathrm{cm}$ pieced as previously reported [20]. Tissue pieces were then subjected to enzymatic dissociation using $0.2 \%$ collagenase in DMEM with 10\% FBS for 90 minutes at $37^{\circ} \mathrm{C}$ and passed sequentially through 18 , 22 , and 25 gauge needles followed by a 40 um cell strainer as reported. The cell suspension was washed twice with DMEM and plated into a T-75 tissue culture flask with growth media consisting of DMEM 10\% EVfree FBS, $1 \%$ penicillin-streptomycin. EVs were also isolated from DU145 and PrEC cell lines. DU-145 cells were plated at $1.5 \times 10^{5}$ cells per T75 flask, and PrECs were plated at $1.5 \times 10^{6}$ cells per $100 \mathrm{~mm}$ plate. Cell cultures were maintained under the previously listed conditions, and after 7 days of culture, or approximately 5 doublings of normal and tumor tissue as previously reported, the medium from the cultured cells was removed and further processed to isolate EVs [20].

The medium for EV isolation was centrifuged at $300 \times$ gravity for 10 minutes at $4^{\circ} \mathrm{C}$. The supernatant was UCF (ultra-centrifuged) at $24,000 \times \mathrm{g}$ for 1 hour at $4^{\circ} \mathrm{C}$. The UCF pellet was resuspended in growth medium and co-cultured (self-culture or cross-culture) with cells for 4-7 days. The UCF pellet was further processed for co-culture (see below) or the isolation of protein for phospho-protein (Kinexus) and Mass Spec analysis. The supernatant from EV isolation was used as conditioned medium (CM) and was concentrated using Amicon Ultra-15 centrifugal filter unit (Millipore, Billerica, MA). The CM was filter sterilized and used for co-culture experiments described below.

\section{Co-culture of prostate tissue extracellular vesicles with} non- and malignant prostate epithelial cell lines

Non-malignant human PrECs and malignant DU145 prostate cells were grown in Lonza Bullet or RPMI medium, respectively supplemented with special additives (PrEC) or $10 \%$ dialyzed EV-free FBS and antibiotics (DU145). The cells were co-cultured with EVs, from normal or malignant prostate tissue. Specifically, PrECs were co-cultured with EVs from prostate tumor tissue and malignant DU145 cells with EVs derived from normal prostate tissue. PrEC cells were also co-cultured for 7 days with CM isolated from DU145 cells.

\section{Soft agar cloning}

Following EV co-culture, cells were grown in normal growth medium. After 7 days, cells were harvested for soft agar colony formation. The lower layer of the dish contained $2 \mathrm{ml}$ of $1 \%$ agarose mixed with growth media; on the top level, $0.4 \%$ agarose mixed with growth media and $0.05-1 \times 105$ cells to a final volume of $1 \mathrm{ml}$. Plates were incubated in $5 \% \mathrm{CO} 2$ at $37^{\circ} \mathrm{C}$ for $2-3$ weeks. Colonies were then counted and images were captured on a Olympus MT2 microscope. Each experiment was analyzed in quadruplicate by 3 different individuals.

\section{Kinex $^{\mathrm{TM}}$ antibody microarray analysis}

The Kinex ${ }^{\mathrm{TM}}$ antibody microarrays are printed in quadruplicate in 32 grids of $8 \times 12$ spots each on glass microscope slide-sized chips with 854 antibodies from over 20 different commercial suppliers. They include 517 pan-specific antibodies for measurement of expressions of 309 protein kinases and 218 other signaling proteins, as well as 337 phospho-site-specific antibodies [21,22]. To perform a Kinex $^{\mathrm{Tx}}$ analysis, extracellular vesicles were harvested as previously discussed and co-culturing was conducted with $1 \times 10^{6}$ cells on $100 \mathrm{~mm}$ plates. Cells were harvested washed twice with PBS, and centrifuged at $14,000 \times \mathrm{g}$ for 5 minutes. Supernatant was discarded and the resulting pellet was frozen at $-20^{\circ} \mathrm{C}$. The lysates with $50 \mathrm{ug}$ protein each from the samples were labeled with the same proprietary fluorescent dye. Each sample was separately applied to opposite sides of the antibody microarray that contains a dam to prevent mixing of the samples. Following incubation of the samples with the Kinex ${ }^{\text {тs }}$ chip, the unbound proteins were washed away and the chips were scanned with a Perkin-Elmer Scan Array Express Reader. Image analysis of the TIF files that were produced was performed with ImaGene 7.0 software from BioDiscovery (El Segundo, CA). Quantification of the signal intensity of all of the detected spots revealed that the difference between duplicates was within $10 \%$ for half of all of the antibodies used.

\section{Protein extraction for Mass Spectrometry analysis}

Protein lysates from PrECs that were co-cultured with patient EVs for 7 days and control PrECs co-cultured 
with PrEC EVs were obtained using a ReadyPrep Sequential Extraction Kit (Bio-Rad) and then the sequential extractions were combined and cleaned up using a ReadyPrep 2-D Clean-Up Kit (Bio-Rad). Total protein concentration was determined using a BCA protein assay kit (Thermo Scientific). Samples were then resolved using NuPAGE SDS-PAGE system (Invitrogen) (4-12\% acrylamide, Bis-Tris with MES SDS Running Buffer) and stained with Gel Code Blue Stain (Thermo Scientific). Gel lanes corresponding to each sample were excised into 3 bands that covered regions of high, medium, and low molecular weight proteins to reduce sample complexity. Each band was then cut into $6 \mathrm{~mm}$ wide pieces and subjected to in-gel tryptic digestion, and then each fraction was washed/dehydrated twice in a 1:1 solution of $0.1 \mathrm{M}$ ammonium bicarbonate (Sigma) and $100 \%$ ACN (Sigma). Disulfide bonds were reduced with $10 \mathrm{mM}$ dithiothreitol (DTT)(Thermo)/0.1 M ammonium bicarbonate for $45 \mathrm{~min}$ at $56^{\circ} \mathrm{C}$ and alkylated with $55 \mathrm{mM}$ iodoacetamide (IAA)(Sigma) for $30 \mathrm{~min}$ at room temperature in the dark, and washed/dehydrated twice as explained above followed by trypsin digestion overnight at $37^{\circ} \mathrm{C}$. After trypsin digestion, peptides were extracted using $25 \mathrm{mM}$ ammonium bicarbonate and $100 \%$ ACN, followed by two rounds of $5 \%$ formic acid and $100 \%$ ACN. The extracts were pooled, dried in a vacuum centrifuge, and stored at $-20^{\circ} \mathrm{C}$ until LC/MS analysis.

\section{Liquid chromatography/ MS analysis of protein digests}

Mass spectrometry analysis was performed at the Rhode Island Hospital Proteomics Core facility by nano-LCESI-MS/MS using an Ultimate3000 nano-LC system (Dionex) controlled with Chromeleon software coupled to a QSTAR XL (Applied Biosystems, Concord, Ontario, CA) mass spectrometer. Tryptic digests were fractionated by reversed-phase chromatography using a C-18 PepMap 100 column $(75 \mu \mathrm{m}$ id $\times 15 \mathrm{~mm}, 3 \mu \mathrm{m}$ particle size, LC Packings/Dionex, Sunnyvale, CA) operating at a flow of $300 \mathrm{~nL} / \mathrm{min}$. A linear separation gradient applied was starting at $5 \%(\mathrm{v} / \mathrm{v}) \mathrm{ACN}$ in $0.1 \%(\mathrm{v} / \mathrm{v})$ formic acid (Buffer A) to $95 \%(\mathrm{v} / \mathrm{v}) \mathrm{ACN}$ in $0.1 \%(\mathrm{v} / \mathrm{v})$ formic acid (Buffer B) over a $40 \mathrm{~min}$ gradient. The column eluate was introduced directly into the mass spectrometer via ESI.

Candidate ions were selected and fragmented using a standard information dependent acquisition (IDA) method. One second MS scans (range between 350 and 1800 Thompson, Thompson $(\mathrm{Th})=\mathrm{Da} / \mathrm{z})$ were used to identify candidates for fragmentation during MS/MS scans. MS/MS scans (2 s; range between 150 and 1800 Th) were collected up to three times after each survey scan. In order for an ion to be considered a candidate for fragmentation it had to be assigned a charge in the range of +2 to +4 .

\section{Data processing for protein identification and quantitation}

Raw LC-MS/MS data were converted using ABSciex MS Data converter software (v1.3 beta) to mgf format for protein identification using MASCOT v2.3.2 search engine (Matrix Science, Boston, MA, USA) by searching against a non-redundant human UniProt database (April 20th, 2012, containing 87,656 protein entries) using the following parameters: tryptic peptides with up to two missed cleavage sites, peptide tolerance of $0.2 \mathrm{Da}$, fragment tolerance of $0.5 \mathrm{Da}$, instrument type: ESI-QUADTOF, and variable modifications: methionine oxidation.

For label-free protein quantitation and proteome comparisons, raw files were converted to mzXML format using ABSciex MS Data converter software (v1.3 beta) and uploaded along with Mascot search results in .dat format into ProteoIQ software (v.2.3.08 BIOINQUIRE Athens, GA, USA). Spectral counting and relative intensity quantification were performed using precursor ion intensities, with the following parameters: mass tolerance of $20 \mathrm{ppm}$, minimum peptide length of 6 amino acids, protein probability of 0.5 , and peptide probability of 0.05 . After protein set generation, the proteins were further filtered using a 0.9 protein probability and normalized according to the number of spectra in each sample. Then the proteins which the 5 Gleason grade 8 patients had in common were placed in a new protein set and were filtered using GO annotations which describe the role of a given gene in a biological process, its molecular function, and cellular component. The GO terms which were selected to filter the results are related to apoptosis, inflammation, immune response, DNA transcription, and DNA translation in order to determine the importance and behavior of these proteins in apoptotic and cell survival pathways.

Ingenuity Pathway Analysis (IPA) (Ingenuity Systems, Redwood City, CA, USA) was used to identify protein networks according to biological functions and/or diseases in the Ingenuity Pathway Knowledge Base (IPKB). The protein accession numbers and the corresponding $\log _{2}$ relative expression values were uploaded into IPA, where the $\log _{2}$ relative expression values are converted to fold change values by the software. Then using these fold change values (with a cutoff of 1.5 for up- or down- regulation) for each protein, IPA determines the statistically relevant $(\mathrm{p}<0.05)$ canonical pathways and functions related to the proteins in each sample. Each pathway and function is assigned a $-\log$ (p-value) that is determined by the number of proteins present in the specific pathway or function and the statistical significance of the expression level of the protein.

\section{Statistical methods}

All cell culture experiments were repeated at least 3 times, unless indicated otherwise, and paired t-tests were used to determine statistical significance. 


\section{Results}

Extracellular vesicle-mediated reversal of drug resistance in prostate cancer

Chemotherapy is currently the major treatment option for castration-resistance prostate cancer. However, chemoresistance is inherent in half of all patients that receive chemotherapy, and the decline of sensitivity to therapeutic agents in patients that initially respond is inevitable [23]. Multiple cellular pathways involving apoptosis, inflammation, angiogenesis, signaling intermediaries, drug efflux pumps, and tubulin are implicated in the development of chemoresistance [23]. It has been shown that resistance to CPT in DU145 cells is due, in part, to expression of Raf kinase inhibitor protein (RKIP) [18]. We hypothesized that in addition to RKIP, resistance to CPT may be due to the release of EVs.

To investigate the effects of EV-mediated transfer of chemoresistance in prostate cancer, we studied the mechanism of resistance to camptothecin (CPT) in human prostate cancer cell lines. The DU145 cell line, a human prostate carcinoma cell line, undergoes extensive apoptosis when treated with 9-nitrocamptothecin (9NC) [18].
CPT inhibits topoisomerase I, thereby inducing singlestrand breaks into the DNA molecule [24]. Conditioned media from parental DU145 cells and DU145 cells resistant to CPT (RC1 cells) were collected, ultracentrifuged, and EVs were collected for co-culture [20]. EVs isolated from DU145 cells were co-cultured with RC1 cells and EVs from RC1 cells were co-cultured with DU145 cells. After 6 days, both groups were treated with CPT, cells were harvested and analyzed for apoptosis via Poly ADP Ribose Polymerase (PARP) cleavage. Upon DNA damage PARP signals DNA repair enzymes. Treatment of DU145 with CPT indicates an increase in PARP cleavage compared to untreated control cells (CTR) indicating PARP mediated activation of DNA repair while DU145 cells cocultured with EVs derived from RC1 cells demonstrated reduction in PARP cleavage similar to the control (Figure 1A, B). These results demonstrate EV-mediated chemoresistance of DU145 cells. DU145 cells co-cultured with RC1 EVs did not undergo apoptosis after CPT treatment, whereas the RC1 cells co-cultured with the DU145 EVs were now sensitized to the apoptosis-inducing effects of CPT (Figure 1C, D). The same experiment was repeated
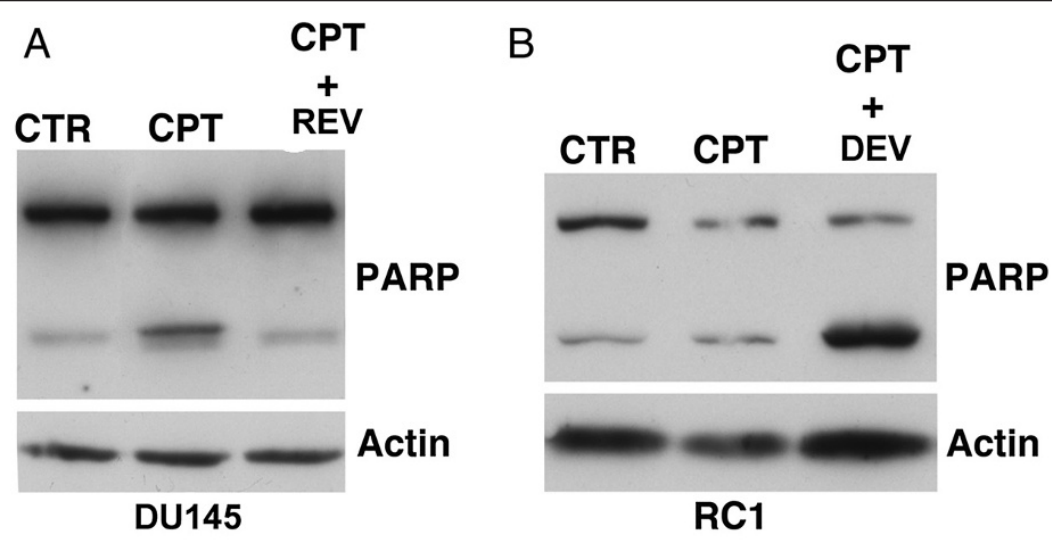

C

$\mathrm{D}$
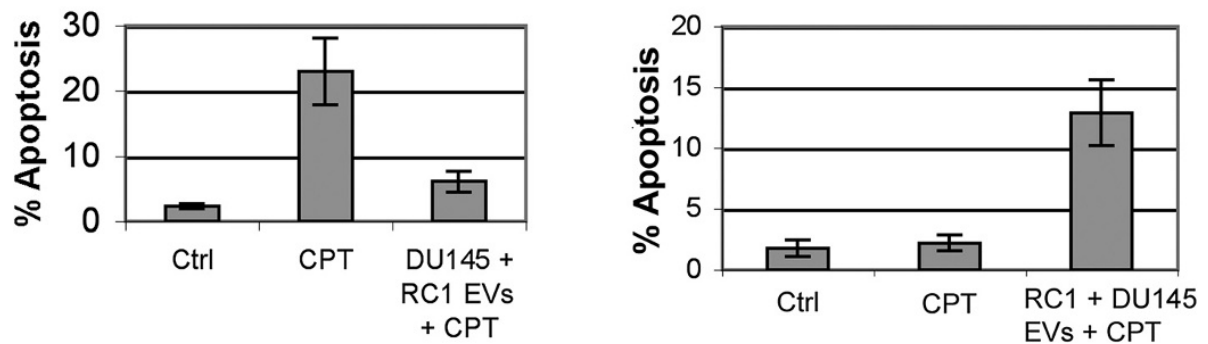

Figure 1 Extracellular vesicle-mediated reversal of apoptosis resistance and sensitivity. A, B. EVs were isolated from DU145 and RC1 cells. The EVs were resuspended in PBS. DU145 cells were co-cultured with RC1 EVs (REV) and RC1 cells were co-cultured with DU145 EVs (DEV). NonEV and EV co-cultured cells were treated with $100 \mathrm{nM}$ CPT for $24 \mathrm{~h}$ and examined for PARP cleavage and actin via Western blot analysis. Proteins (unless indicated $50 \mathrm{\mu g} / \mathrm{sample}$ was used for Western blot analysis) were separated by 10\% SDS-PAGE, transferred to nitrocellulose and analyzed with antibodies to the indicated proteins. C,D. The same experiment from $\mathbf{B}, \mathbf{C}$ was repeated and the samples were examined for apoptosis via propidium iodide staining using a flow cytometer. The data is the mean $+/-$ s.d. of 2 independent experiments performed in triplicate. Note: for all Western blots described in this Figure legend and for all other subsequent Figure legends, the exposure time used to identify the various proteins was variable. 
and the cells were analyzed for apoptosis via flow cytometry. As noted in Figure 1C, DU145 cells became resistant to CPT-induced apoptosis after co-culture with RC1 EVs. Conversely, RC1 cells underwent apoptosis after being cocultured with DU145 cells EVs and then treated with CPT (Figure 1D). These results indicate a phenotypic shift facilitated by the co-culturing with EVs.

\section{Extracellular vesicle-mediated reversal of malignant prostate cancer phenotype}

To better understand the phenotypic switching capacity of EVs, several strategies were applied. One of the hallmarks of malignant transformation of cells is the ability to exhibit anchorage-independent growth [25]. Therefore, we continued to examine EV-mediated phenotype changes using a non-malignant model of prostate cancer in a malignant cell line to see if the phenotype can be transferred as measured by soft agar colony formation. EVs were harvested from a malignant human prostate cancer cell line (DU145), as well as from an immortalized, non-tumorigenic prostate epithelial cell line (PrEC cells), and were collected for co-culture for vesicle characterization. EVs isolated from DU145 cells were co-cultured with PrECs and EVs from PrEC cells were co-cultured with DU145 cells. The number of EVs used for co-culture was normalized by counting the total number of EVs within a particular size of 30-1000 nm using the NanoSight NS500 (NanoSight, Wiltshire United Kingdom).

After 7 days in culture, we measured the ability of each experimental condition (from above) to display anchorage independent growth in soft agar for 14 days. Since the malignant phenotype includes an increased ability to exhibit anchorage-independent growth, a significant increase or reduction in the number of colonies generated was viewed as a shift towards a tumorigenic phenotype or towards a normal phenotype, respectively. As shown in Figure 2, co-culture of DU145 cells with EVs isolated from PrECs prevented the colony formation in soft agar, indicating that anchorage-independent growth was significantly suppressed $(\mathrm{p}<0.0004)$ in comparison to DU145 cells without EVs (Figure 2A). Remarkably, the reciprocal effect was also observed where significant changes in colony formation and anchorage independent growth in non-tumorigenic PrECs that were co-cultured with EVs isolated from DU145 cells $(\mathrm{p}<0.0003)$ compared to PrECs without EVs (Figure 2A). To determine if the results we observed were a direct effect of EV co-culture with recipient cells or could be recapitulated indirectly with factors (i.e., cytokines, growth factors, etc.) released into the medium, we isolated CM from DU145 cells used for EV purification. As shown in Figure 2B, EVs isolated from DU145 cells stimulated PrEC soft agar colony formation. In contrast, CM isolated from DU145 cells did not significantly change
PrEC soft agar growth (Figure 2B). This indicates that the phenotype shift we observed is a direct effect of DU145 EVs.

\section{Kinexus proteomic antibody array analysis of transferred proteins}

To determine the proteins that are involved in or might be responsible for "phenotypic switching", we utilized Kinexus phospho-protein microarray analysis (Vancouver, BC) in the DU145 cells co-cultured with PrEC EVs. EVs were isolated from PrECs after 7 days in culture as described [20]. PrEC EVs were then co-cultured with DU145 cells for 7 days after which the cells were harvested and the pellet sent to Kinexus Bioinformatics for analysis. The ability of EVs to elicit a phenotypic switch was therefore verified in the proteins that were transferred and analyzed. As a control a sample with DU145 cells co-cultured with DU145 EVs was also generated. Table 1 shows a portion of the results of the microarray analysis indicating the fold change in protein expression ( $\mathrm{Z}$ score ratio).

To further validate these results, we confirmed the proteomic data with Western blot analysis. We performed Western blot analysis with an aliquot of the sample that was retained in the lab prior to the shipment of the samples to Kinexus. We observed a reduction STAT3 expression in DU145 cells co-cultured with selfEVs, in comparison to DU145 cells co-cultured with PrEC EVs. Further, there was a significant increase in SOCS3 expression in the DU145 cells co-cultured with PrEC EVs (Figure 3). It should be noted that we did not observe any difference in the levels of the proteins examined in parental DU145 cells when compared to DU145 cells co-cultured with DU145 EVs (data not shown). Our results implies that aberrant STAT3 signaling may be inhibited by EV release and transfer of SOCS3 or regulators of STAT3 signaling network.

\section{Mass spectrometry analysis of prostate cancer patient derived extracellular vesicles}

We extended our studies on DU145 and PrEC EVs and phenotype shifting to EVs derived from 2 prostate cancer patients both with Gleason grade 8. Soft agar growth was measured in PrECs after co-culture with EVs from prostate cancer patients 18 and 19 . EVs from patients 18 or 19 significantly increased soft agar growth in nonmalignant PrECs ( $<0.0005$ and $\mathrm{p}<0.0001$, respectively) (Figure 4). A portion of the sample used for soft agar cloning was analyzed by mass spectrometry. Table 2 shows a partial list of the proteins identified in PrECs exposed to tumor-derived EVs from patients 18 and 19 as well as the $\log _{2}$ relative expression of each protein. Some 14-3-3 isoforms are associated with increased malignancy and are therapeutic targets [26] and our 

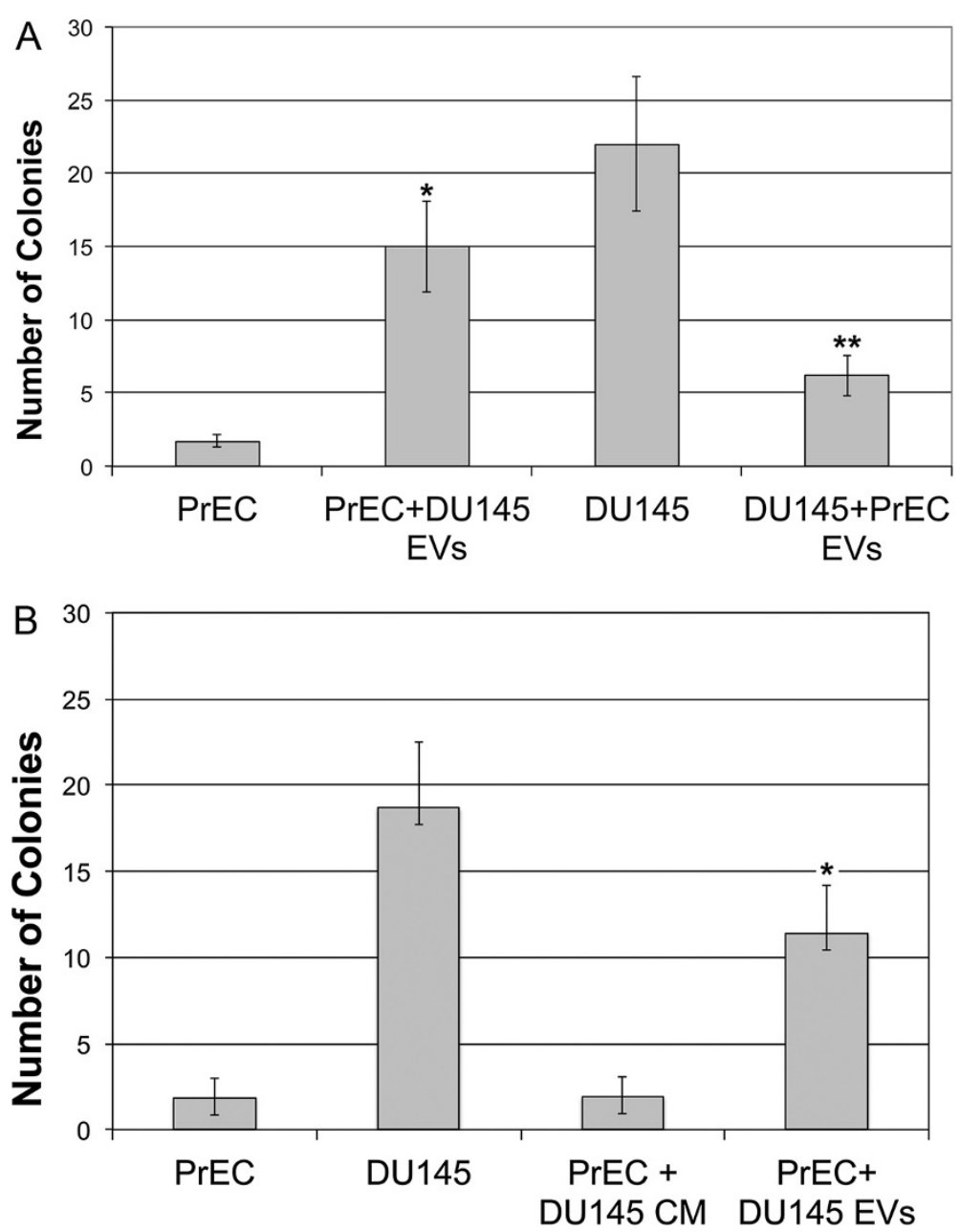

Figure 2 Extracellular vesicle-mediated reversal of soft agar growth. A, EVs were isolated from normal prostate (PrEC) and malignant (DU145) cells. PrECs were co-cultured with DU145 EVs and DU145 cells were co-cultured with PrEC EVs for 7 days. B, The same experiment from A was repeated with EVs and conditioned medium (CM) isolated from DU145 cells and co-cultured with PrEC cells for 7 days. 50 mls of CM was concentrated and used for the experiment. In both experiments, cells were harvested and utilized for soft agar cloning. Soft agar cloning was examined using $0.7 \%$ agarose in PBS and mixed with $1 \times$ media with $10 \%$ FBS. The top layer consisted of $0.35 \%$ agarose in PBS, $1 \times$ media with $10 \% \mathrm{FBS}$, and $1 \times 105$ cells per dish. Dishes were incubated at $37^{\circ} \mathrm{C}$ and $5 \% \mathrm{CO}$. After two weeks, cell colonies were counted. 5 fields/dish using the $40 \times$ objective were counted and there were 5 dishes/condition. A paired t-test was performed to analyze the increase in soft agar colony formation of in A: PrEC + DU145 EVs when compared to untreated PrEC cells, ${ }^{*} p<0.002$, and the decrease in soft agar colony formation of DU $145+$ PrEC EVs when compared to untreated DU145 cells ${ }^{* *} p<0.0004$. In B: PrEC $+p<0.0003$ when compared to untreated PrEC cells. ${ }^{*} p<0.0005$.

analysis revealed an increase of 14-3-3 zeta/delta which was confirmed by Western blot analysis (Figure 4). Also of note is the increase in pRKIP when patient 18 and 19 EVs were co-cultured with PrECs in reference to the levels of RKIP in PrECs alone. RKIP has been shown to regulate apoptosis and cell survival in prostate cancer [18]. Western blot analysis revealed that RKIP was phosphorylated after co-culture of patient 18 and $19 \mathrm{EVs}$ with PrECs (Figure 5A). This result would explain, in part, our data in Figure 4 because pRKIP antagonizes the function of RKIP and allows for Raf/MAPK signaling to occur. This pathway promotes oncogenesis and cell proliferation and, presumably, soft agar growth.
In our analysis of the total proteome content of PrECs exposed to EVs derived from patient 18, we identified 36 protein groups in PrECs alone and 44 protein groups in PrECs with Patient 18 EVs. From these, 8 protein groups were unique to PrECs and 16 were unique in Patient 18 EVs with 28 common protein groups (Figure 5B). Exposure of PrECs with EVs from Patient 19 yielded similar results (Figure 5C). For example, Macrophage migration inhibitory factor (Uniprot ID: MIF_HUMAN) and Peptidyl-prolyl cis-trans isomerase A (Uniprot ID: PPIA_HUMAN) were found to be unique in both Patient $18 \mathrm{EVs}$ and Patient $19 \mathrm{EVs}$ when compared to PrECs alone. Analysis of proteome content between 
Table 1 EV-mediated transfer of proteins via Kinexus phospho-protein microarray analysis in DU145 cells co-cultured with PrEC EVs

\begin{tabular}{lc}
\hline Target protein name & Z-ratio (DuP, Du) \\
\hline PKCZ & 1.42 \\
SOCS3 & 1.30 \\
PKCm (PKD) & 1.05 \\
KAP & 1.05 \\
STAT5A & 1.04 \\
Cyclin G1 & 1.00 \\
IKKa & -1.01 \\
P38g MAPK (Erk6) & -1.02 \\
CK1g & -1.03 \\
MEK5 (MAP2K5) & -1.08 \\
CDK1 (CDC2) & -1.09 \\
STAT6 & -1.09 \\
IKKa & -1.14 \\
RIPK1 & -1.25 \\
STAT3 & -1.48 \\
PAK3 & -1.52 \\
RSK1 & -3.43 \\
\hline EXFacella &
\end{tabular}

Extracellular vesicles were isolated from PrECs after 7 days in culture as described. PrEC EVs were then co-cultured with DU145 cells for 7 days after which cells were harvested and the harvested cell pellet sent to Kinexus Corporation, Vancouver, BC for analysis. As a control, a sample with DU145 cells co-cultured with DU145 EVs was also generated. These are a portion the results of the array analysis indicating the fold change in protein expression (Z score) of DU145 cells co-cultured with PrEC EVs when compared to DU145 cells co-cultured with DU145 EVs.

patients 18 and 19 yielded minimal differences between the numbers of protein groups identified in each sample indicating low patient heterogeneity (Figure 5D).

We examined the EV content of 3 additional Gleason grade 8 patients (Patients 13, 14, and 16) (Figure 6). The Venn diagram shows that there are 222 common proteins between these patients. The bar graph shows the functionalities listed by IPA based on the ProteoIQ protein relative expression values. The relative expression value of each protein in addition to the presence or absence of key proteins associated with that term both contribute to the significance value assigned to each function or pathway (indicated by the threshold line across the bar graph in Figure 6). The terms presented in the bar graphs were selected because they are related to cancer and/or are important topics discussed in the paper.

In addition, we compared patients 18 and 19, with patients 13,14 , and 16 , and determined the common proteins between these 5 Gleason grade 8 patients. These 71 common proteins between the 5 Gleason grade 8 patients were then further filtered according to GO annotations which are related to apoptotic and cell survival

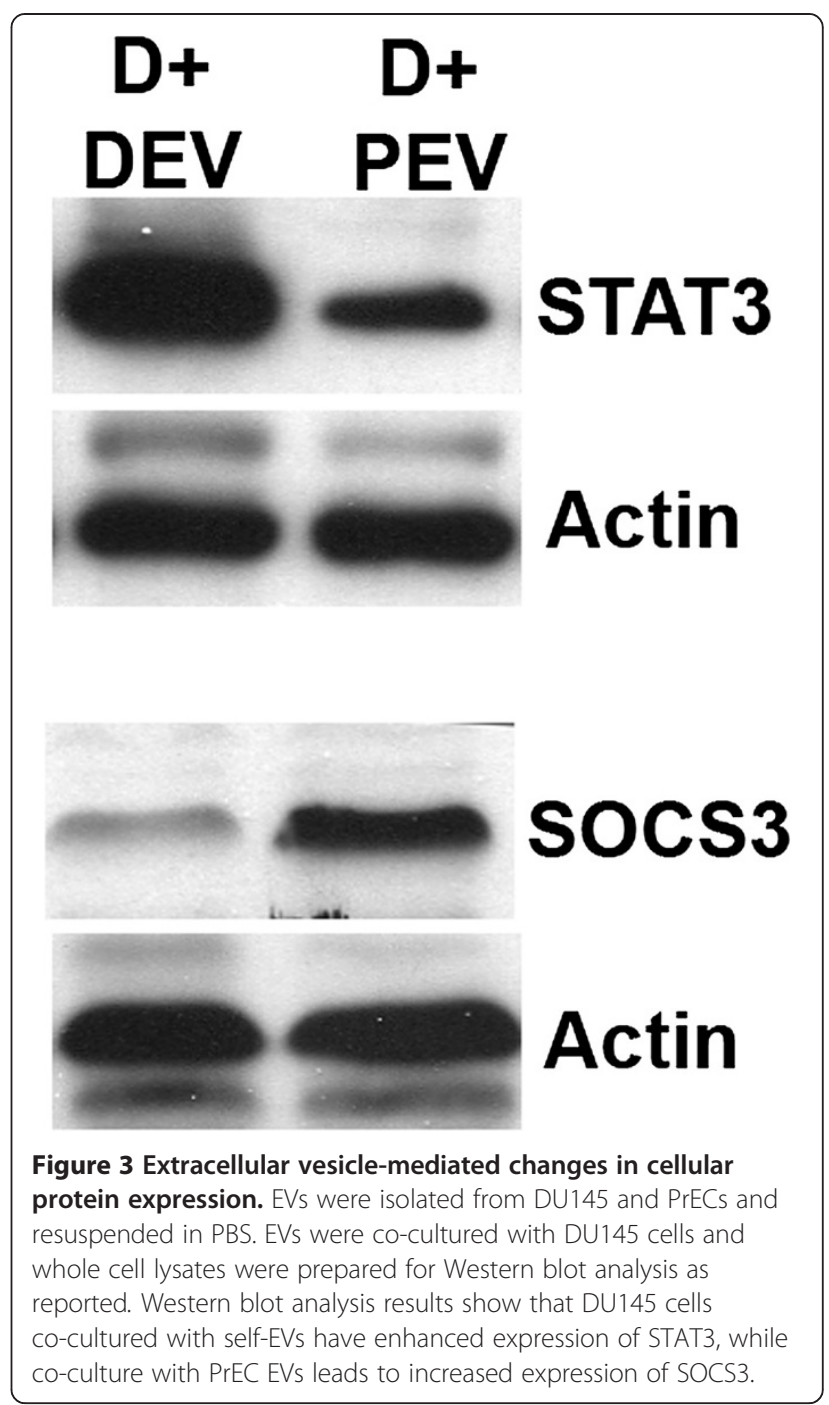

pathways (seen in Tables 3, 4, 5, and 6). Some of the common proteins included: Prohibitin (Uniprot ID: PHB_HUMAN), 14-3-3 (Uniprot ID: 1433E_HUMAN), and Annexin A1 (Uniprot ID: ANXA1_HUMAN). The importance of the proteins found in these tables is that we found proteins across all patients that are associated with apoptosis, growth and proliferation, inflammation, immune response, and DNA transcription and translation. Again with all 5 Gleason grade 8 patients was detected that 14-3-3 zeta/delta/eta was a protein in common with all 5 patients (Table 2 and Additional file 1: Table S1). This proves that there is level of homogeneity in protein content amongst these 5 Gleason 8 patients, which provides a basis for targeting these proteins to improve therapeutic methods.

We also analyzed this group of 71 proteins using IPA to determine whether the interaction of these proteins is similar across all of the patients. IPA showed that there is similarity in the level of significance of functions and 


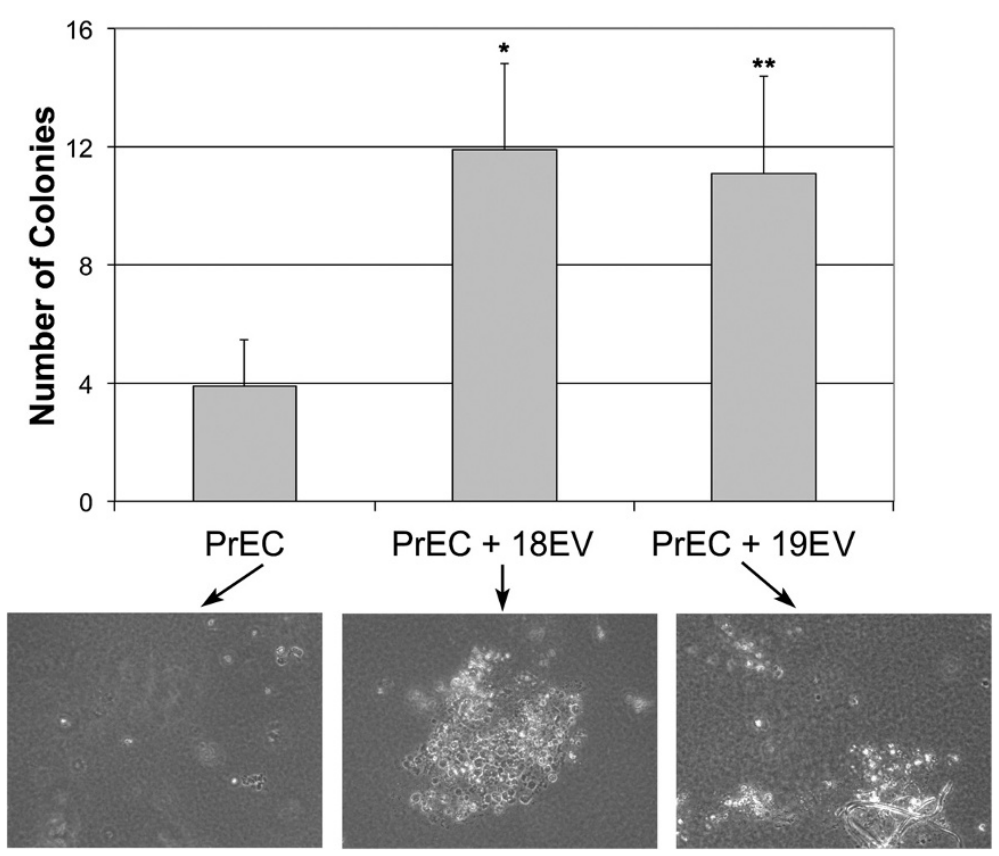

Figure 4 Enhancement of soft agar growth via prostate patient-derived EVs. EVs were isolated from 2 prostate cancer patients with Gleasons grade 8. The EVs were co-cultured with PrECs for 7 days after which soft agar growth was determined. 6 fields/dish were counted and the data represents the mean $+/-$ s.d. of 2 independent experiments performed in triplicate. A paired t-test was performed to analyze the increase in soft agar colony formation of PrEC cells when co-cultured with EVs from patient $18,{ }^{*} p<0.0005$, and patient $19{ }^{* *} p<0.0001$ when compared to untreated PrEC cells. Note the increase in colony size in PrECs (bottom panel) co-cultured patient tumor EVs. The pictures are representative of an area of a field that was counted.

canonical pathways related to apoptosis and cell survival across all the patients. The data sets from the 5 patients were compared using a Comparison Analysis Tool in the IPA software. The p-value, in this case, is the measure of the likelihood that the association between a set of genes in the dataset and a related function or pathway is due to random association. The cutoff value for the bar graph is set at $\mathrm{p}<0.05$ (or $-\log <1.3$ ) as indicated by the threshold line in Figure 7. So all the functions across all the patients show a statistically significant non-random association, and the majority of the pathways across all the patients also show a statistically significant nonrandom association. The fact that some of the canonical pathways are statistically significant in some patients but not in others can be attributed to some level of heterogeneity across patients. But for the majority of the functions and canonical pathways, the level of homogeneity in protein content and function and pathway significance can clearly be seen and can definitely prove to be a useful tool in the future development of targeted cancer therapeutics.

\section{Discussion}

The results of this study provide direct evidence of the therapeutic potential of EVs in prostate cancer. The phenotypic changes derived by EV co-culture with

Table 2 Comparison of relative protein expression between PrECs alone and PrECs co-cultured with patient EVs

\begin{tabular}{|c|c|c|c|c|c|c|c|}
\hline \multirow[t]{2}{*}{ Accession \# } & \multirow[t]{2}{*}{ Protein name } & \multicolumn{2}{|c|}{ PrEC vs. PrEC + P18 EVs } & \multicolumn{2}{|c|}{ PrEC vs. PrEC + P19 EVs } & \multicolumn{2}{|c|}{ PrEC + P18 EVs vs. PrEC + P19 EVs } \\
\hline & & $\log _{2}$ & \# Pep & $\log _{2}$ & \# Pep & $\log _{2}$ & \# Pep \\
\hline P62258 & 14-3-3 protein zeta & -0.64 & 2 & 0.59 & 2 & 1.23 & 2 \\
\hline P06733 & Alpha-enolase & 0.051 & 5 & 0.044 & 2 & -0.007 & 2 \\
\hline P04083 & Annexin A1 & 0.47 & 9 & 0.69 & 10 & 0.22 & 10 \\
\hline P00403 & Cytochrome c oxidase subunit 2 & 0.86 & 4 & 0.82 & 4 & -0.04 & 4 \\
\hline P68104 & Elongation factor 1-alpha 1 & 0.04 & 8 & 0.39 & 8 & 0.35 & 8 \\
\hline P30086 & PEBP1 (RKIP) & 1.12 & 5 & 1.88 & 4 & 0.75 & 4 \\
\hline P35232 & Prohibitin & 0.08 & 2 & 0.41 & 2 & 0.33 & 2 \\
\hline
\end{tabular}




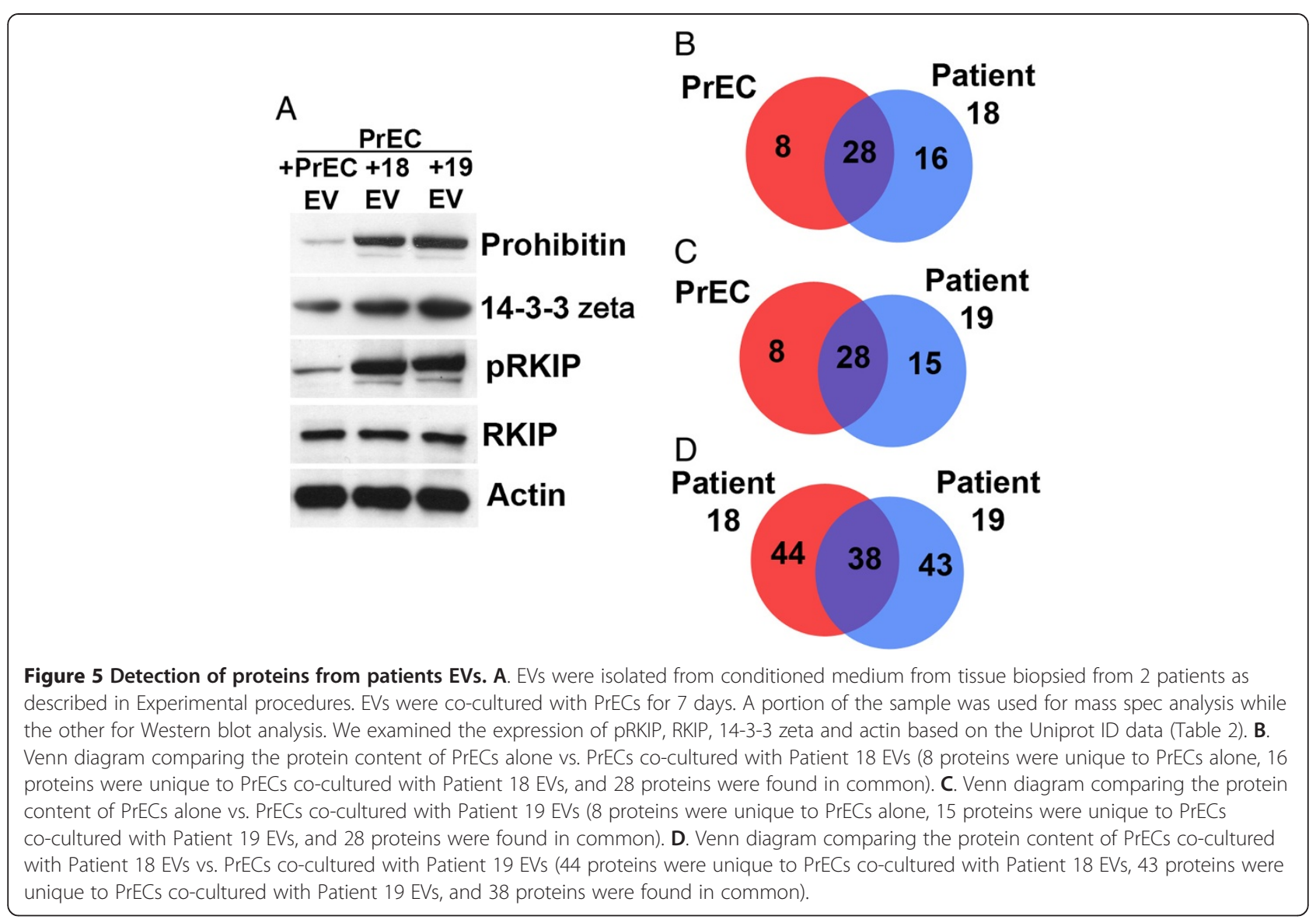

normal or malignant prostate cells demonstrate the potential of EVs in the horizontal transfer of proteins. This can provide a potential opportunity for the development of new diagnostic and/or therapeutic measures. By manipulating the uptake, incorporation, and expression of exogenous material from EVs may results in phenotype switching. Horizontal gene transfer is understood to include any mechanism where genetic material is exchanged from non-parent donors to recipient cells. These methods include cell-cell interactions, such as cell fusion, and cellular interactions with mobile genetic elements, including plasmids and bacteriophages [27]. Other methods of horizontal gene transfer have been implemented in the studies of tumor progression. Gaiffe et al. illustrated that apoptotic cells can serve as DNA vectors through an endocytic process where apoptotic cells are internalized by non-specialized cells [28]. As successful gene transfer depends on in situ signaling, recognition, molecular triggers, and the surrounding environment, it is surprising that such unlikely elements can drive phenotype-switching, and consequently tumor progression.

In this manuscript we show that the phenotypes associated with malignant transformation and normal cell growth in prostate cells, as well as chemo-resistance/ -sensitivity, can be transferred by EVs and via biopsied EVs isolated from patient tumor cells. Seemingly, exposing cells of a normal prostate phenotype with EVs from a malignant phenotype, and vice versa, leads to a transfer of biological materials between phenotypes, and therefore triggers genetic changes within the cells. Our results demonstrate that the cancer phenotype in prostate cells can be reversed or transferred via EVs. The transfer of genetic material derived from non-malignant prostate cells via EVs to a malignant cell line in vitro seemingly reversed the malignant transformation of the prostate cells. One unanswered question is whether the reciprocal events are occurring; if malignant-derived EVs transferred to normal cells fully facilitate a change to malignant phenotype, or if they are limited to promotion of specific hallmarks of malignant transformation. Although EVs from explant tissues (patient tissue samples) significantly increased soft agar growth in normal PrECs and malignant DU145 prostate cells, demonstrating an EV-mediated promotion of the malignant phenotype, our results are insufficient to conclude that this promotion would lead to tumorigenesis in vivo.

Notably, the observed genetic changes resulting from the EV-mediated, horizontal gene transfer included alterations in the expression of several clinically relevant 
A

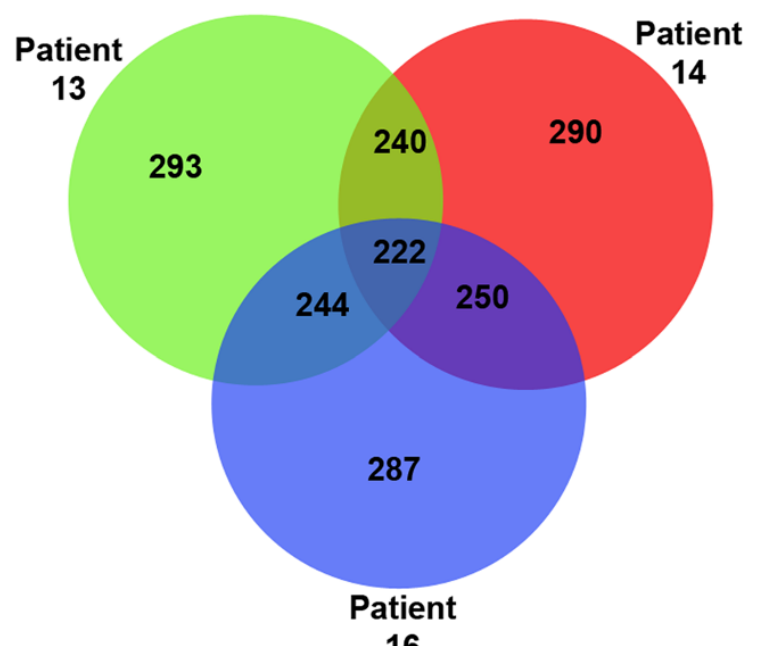

16

B

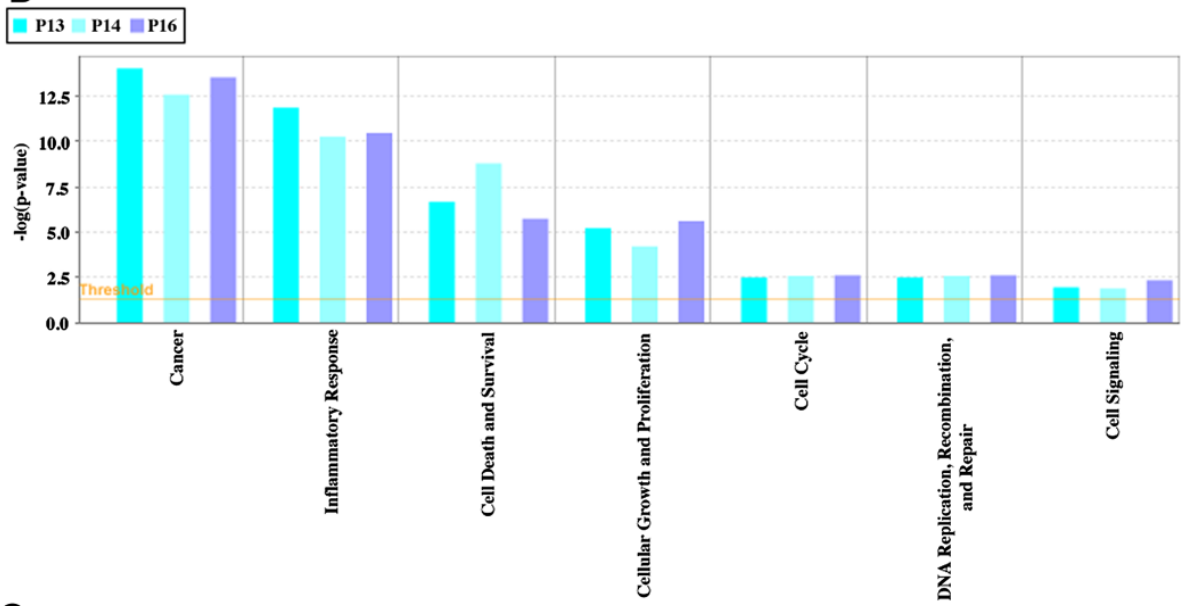

C

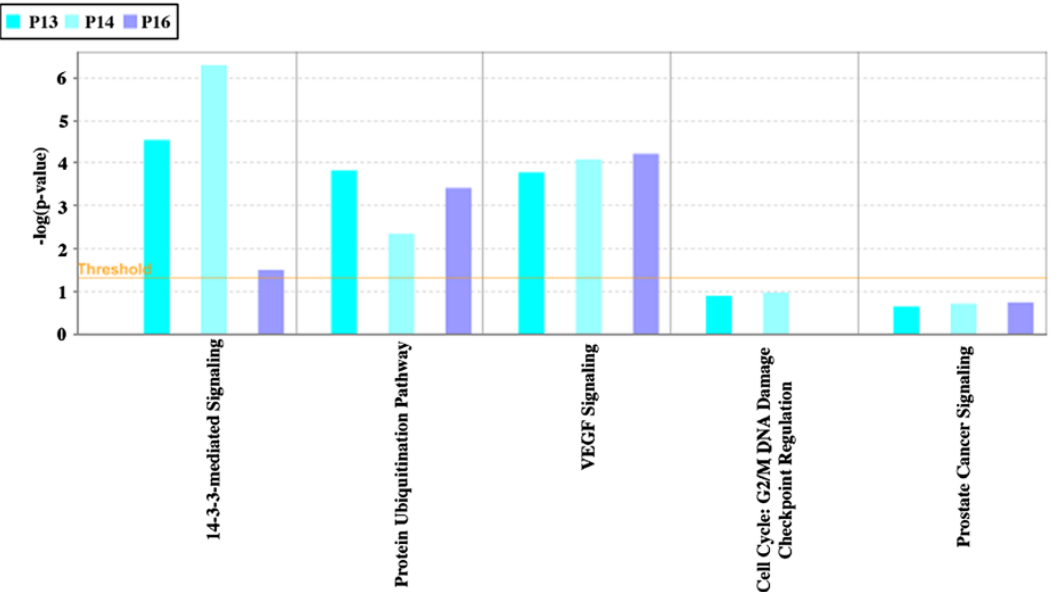

Figure 6 Common proteins between patients 13, 14, and 16 and associated functions and canonical pathways. The Venn diagram shows the common and unique proteins between patients 13, 14, and 16, A The bar graphs show the significance $(-\log (p-v a l u e))$ of specific functions, $\mathbf{B}$, and canonical pathways, $\mathbf{C}$, in each patient. The threshold cutoff of significance is $p<0.05$ (or $-\log =1.3$ ).

proteins, such as RKIP. Previous studies have shown the correlation between the expression levels of RKIP and tumorigenicity in prostate cancer cells [18]. RKIP is required for human cancer cells to undergo druginduced apoptosis, and it suppresses metastasis in prostate cancer. In regard to chemosensitivity, Chatterjee 
Table 3 Apoptosis related proteins found in the common proteins between patients 13, 14, 16, 18, and 19

\begin{tabular}{ll}
\hline Accession \# & Protein name \\
\hline P62258 & 14-3-3 protein epsilon \\
P63104 & 14-3-3 protein zeta/delta \\
P10809 & $60 \mathrm{kDa}$ heat shock protein, mitochondrial \\
P11021 & $78 \mathrm{kDa}$ glucose-regulated protein \\
O43707 & Alpha-actinin-4 \\
P04083 & Annexin A1 \\
P08758 & Annexin A5 \\
P27797 & Calreticulin \\
P14625 & Endoplasmin \\
P04406 & Glyceraldehyde-3-phosphate dehydrogenase \\
P32119 & Peroxiredoxin-2 \\
P02545 & Prelamin-A/C \\
P35232 & Prohibitin \\
P14618 & Pyruvate kinase isozymes M1/M2 \\
P08670 & Vimentin \\
P21796 & Voltage-dependent anion-selective channel protein 1 \\
P13010 & X-ray repair cross-complementing protein 5 \\
\hline
\end{tabular}

Using Gene Ontology (GO) terms, the common proteins between patients 13, $14,16,18$, and 19 were filtered according to GO terms which are related to apoptosis.

et al. showed that a rapid up-regulation of RKIP triggers apoptosis during chemotherapy treatment in drugsensitive human prostate cells, but does not in drugresistant cells [18]. Maximal cellular expression levels of RKIP correlated perfectly with the onset of apoptosis in chemosensitive cells, but in cells that were resistant to DNA-damaging agents, treatment with such drugs did not up-regulate RKIP expression.

We confirmed that RKIP plays a similar role in prostate tumorigenesis under our experimental conditions through assessing levels of the protein expression via mass spec. Our results indeed provided evidence that in addition to its roles in metastatic capacity and intracellular, chemotherapymediated apoptosis in prostate cancer cell lines, RKIP affects the chemosensitivity of prostate cells. Through packaging in EVs or by the transfer of factors that enhance protein

Table 4 Inflammation and immune response related proteins found in the common proteins between patients $13,14,16,18$, and 19

\begin{tabular}{ll}
\hline Accession \# & Protein name \\
\hline P10809 & 60 kDa heat shock protein, mitochondrial \\
P04083 & Annexin A1 \\
P08238 & Heat shock protein HSP 90-beta \\
P32119 & Peroxiredoxin-2 \\
\hline
\end{tabular}

Using Gene Ontology (GO) terms, the common proteins between patients 13, $14,16,18$, and 19 were filtered according to $\mathrm{GO}$ terms which are related to inflammation and immune response.
Table 5 Growth and proliferation related proteins found in the common proteins between patients $13,14,16,18$, and 19

\begin{tabular}{ll}
\hline Accession \# & Protein name \\
\hline P62258 & 14-3-3 protein epsilon \\
Q04917 & 14-3-3 protein eta \\
P11021 & 78 kDa glucose-regulated protein \\
P04083 & Annexin A1 \\
P07355 & Annexin A2 \\
P06576 & ATP synthase subunit beta, mitochondrial \\
P27797 & Calreticulin \\
Q00610 & Clathrin heavy chain 1 \\
Q06830 & Peroxiredoxin-1 \\
P35232 & Prohibitin \\
P13010 & X-ray repair cross-complementing protein 5 \\
\hline Using Gene Ontology (GO) terms, the common proteins between patients 13, \\
14, 16, 18, and 19 were filtered according to GO terms which are related to \\
the growth and proliferation of cells.
\end{tabular}

production, RKIP expression is induced or suppressed leading to chemosensitivity or chemoresisitance, respectively. An increase in RKIP was observed from mass spec analysis (Table 2) from patient samples co-cultured with PrECs. Thus, the RKIP that is packaged in EVs provides another chemotherapy or resistance mechanism that can be targeted. Expression levels of RKIP are predictive of clinical outcome as the level of metastasis in prostate cancer decreases as the levels of RKIP expression increases. Through utilizing EVs to modify the expression levels of RKIP in prostate cancer, it is possible that the metastatic potential of tumor cells can be targeted by an EV-mediated induction of RKIP. Further,

Table 6 DNA transcription and translation related proteins found in the common proteins between patients 13,14 , 16,18 , and 19

\begin{tabular}{ll}
\hline Accession \# & Protein name \\
\hline Q04917 & 14-3-3 protein zeta \\
P63104 & 14-3-3 protein zeta/delta \\
P06733 & Alpha-enolase \\
P27824 & Calnexin \\
P27797 & Calreticulin \\
P04843 & Dolichyl-diphosphooligosaccharide-protein \\
& glycosyltransferase subunit 1 \\
P68104 & Elongation factor 1-alpha 1 \\
P11142 & Heat shock cognate 71 kDa protein \\
P32119 & Peroxiredoxin-2 \\
P35232 & Prohibitin \\
P68371 & Tubulin beta-4B chain \\
P13010 & X-ray repair cross-complementing protein 5
\end{tabular}

Using Gene Ontology (GO) terms, the common proteins between patients 13, $14,16,18$, and 19 were filtered according to GO terms which are related to DNA transcription and translation. 


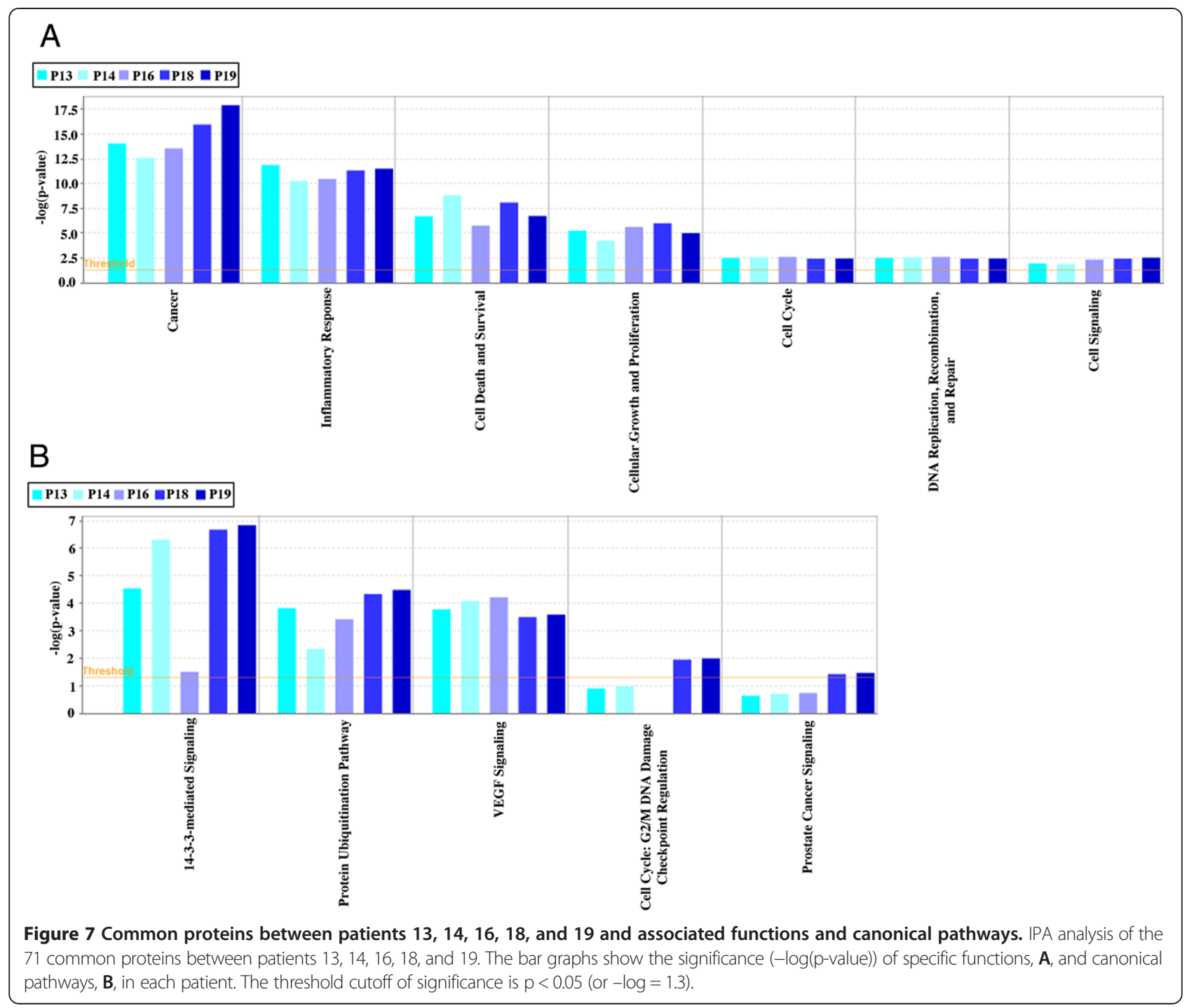

such implementation of EVs as a therapeutic strategy for prostate cancer would likely also lead to apoptosis in target cells. These findings suggest an additional role for RKIP in tumorigenesis.

Our results further indicate that the in vitro recipient cells of horizontally transferred genetic material, via EV coculture, have altered expression levels of STAT3, and SOCS3. Signal transducer and activator of transcription 3 (STAT3) is a pro-survival protein responsible for the upregulation of proteins that promote cell proliferation and growth $[29,30]$. During tumor development and subsequent metastatic progression, the ability of tumors to invade the lymphovascular system is mediated by STAT3 [31]. STAT proteins are substrates for the proto-oncogene c-Src, and can mediate c-Src's biologic effects, which include androgen-induced proliferation of prostate cancer and potentially even the transition to androgen-independent growth [32,33].
Src is highly expressed in prostate cancer cell lines as well as in the majority of prostate cancer specimens [32]. Maintenance of cancer cell proliferation and survival is mediated by STAT3 even after sustained c-Src inhibition through the activation of pro-survival genes, [34]. Hence, STAT3 has been identified as an attractive target for prostate cancer, where its activity is inherently activated. Inhibition of STAT3, though disruption in activation or expression, leads to cessation of tumor cell growth and apoptosis [29]. To date, chemotherapeutic agents such as CPT have been demonstrated to down-regulate STAT3 levels. Our data suggests that EVs are immune to these limitations and can effectively eliminate the effects of STAT3 mediated signaling in prostate cancer. As shown in Figure 3, levels of STAT3 are down-regulated, and levels of SOC3 are upregulated after the malignant DU145 cells are co-cultured with non-malignant PrEC EVs (also shown in Table 1). Suppressor of cytokine signaling 3 (SOCS3) plays a negative 
regulatory role in the STAT3 signaling pathway, and overexpression of the protein is adequate to prevent STAT3 activation [34]. The suppression of STAT3 suggests an endogenous epigenetic mechanism that normal, nonmalignant cells produce to suppress STAT3-mediated cell survival signaling.

Utilizing 2 patients with Gleason grade 8 indicated, via mass spectrometry analysis, the enhanced RKIP and prohibitin was upregulated to non-malignant PrECs (Table 2). pRKIP is an inhibitor of RKIP function [35] and allows Raf-mediated cancer cell survival to be enhanced. Prohibitin was originally identified as a putative tumor suppressor protein that enhances p53 transcription [36-38]. A recent study has demonstrated that prohibitin proteins are necessary for the proliferation of cancer cells [39]. Thus the induction/phosphorylation of these proteins via EV-mediated transfer would promote the change, a switch in phenotype to promote prostate cell survival. Indeed our results from Figure 4 demonstrate that PrECs have acquired the ability to significantly grow in soft agar $(\mathrm{p}<0.0005$ and 0.0001$)$ when co-cultured independently with 2 Gleason grade 8 patients EVs. Our results provide preliminary insight into putative mechanisms leading to phenotype switching of normal tissue via EVs released from malignant adjacent tissue.

14-3-3 proteins are a family of conserved regulatory molecules expressed in all eukaryotic cells. 14-3-3 proteins have the ability to bind a multitude of functionally diverse signaling proteins, including kinases, phosphatases, and transmembrane receptors. It has been demonstrated that expression of 14-3-3 proteins is significantly elevated in multiple cancers $[40,41]$. Our proteomic and IPA analysis determined the enhancement of 14-3-3 zeta/delta expression in our co-culture of patients 18 , and 19 EVs with PrEC cells (Figure 5). In addition, these proteins along with 14-3-3 epsilon was determined to be a common protein found between patients 13,14 and 16 (Tables 3, 4 and 5). In prostate cancer models, the inhibition of PC3M cell growth was abrogated, in part, by the suppression of 14-3-3 zeta/delta [42]. It has been shown that 14-3-3zeta is an androgen-responsive gene that activates proliferation, cell survival, and androgen receptor transcriptional activity and facilitate the progression of prostate cancer [43]. Similarly, 14-3-3eta has been shown to enhance androgen- and mitogen-induced androgen receptor transcriptional activity in castrationrecurrent prostate cancer [44]. In contrast, 14-3-3 $\sigma$ expression is down-regulated during the neoplastic transition of prostate epithelial cells [45] while there is epigenetic inactivation of $14-3-3 \sigma$ occurs at an early stage of prostate tumor development [46]. 14-3-3 proteins complex with many signaling molecules, including the Raf-1 kinase [47]. 14-3-3 beta and zeta can be associated with Raf in mammalian cells and accompany Raf to the cell membrane. Therefore, 14-3-3 proteins may participate in or be required for the regulation of Raf function [48]. Raf A, B, and C activity is differentially regulated by its $\mathrm{C}$-terminal and internal 14-3-3 binding domain. In this model, prohibitin, acting as scaffold protein, affected C-RAF activation in a stimulatory manner and interfered with the internal 14-3-3 binding site in CRAF [49]. Prohibitin-mediated activation of C-Raf at membranes occurs by direct 14-3-3 displacement most probably from the internal 14-3-3 binding site [49]. We observe enhanced levels of prohibitin and 14-3-3 zeta/ delta in PrEC cells after co-culture with patient 18 and 19 EVs (Figure 5). Although prohibitin may displace 143-3 zeta/delta from binding to Raf, 14-3-3 zeta/delta could still stimulate androgen receptor-mediated growth and prohibitin Raf-mediated MAPK signaling promoting oncogenic growth of PrEC cells. Our results suggest that 14-3-3 proteins may provide a therapeutic target in prostate cancer patients.

\section{Conclusions}

It is highly feasible that we could target tumor progression through utilizing EVs as a therapeutic agent or tumor biomarker. The presence of EVs in cancer seemingly aids in accelerating the genetic changes necessary for tumor progression. Thus, probing the relationships between the maintenance of normal and malignant states and the import/export of proteins holds promise towards unveiling the potential diagnostic and/or predictive nature of EVs in cancer. Indeed, our detection of 14-3-3 proteins and their subsequent enhanced protein levels mediated by patient EVs provide a potential therapeutic target. Our intent is to establish EVs as indicators of therapeutic effectiveness, disease recurrence or resistance, and/or possible metastases. We are continuing our studies to identify the content of EVs derived from normal and malignant prostate tissue, using the same in vitro experimental approaches. We are expanding the database from which we will obtain EVs to include different grades of prostate tumor specimens from a racially and ethnically diverse population of men. Additional proteomic analysis should produce a comprehensive database of the proteins associated with EV transfer including prostate-specific trafficking genes, tumor cell surface markers, and other proteins that are specifically associated with the pathogenesis of prostate cancer. Despite the unresolved questions surrounding extracellular vesicle density, content, and transport of biological material cells between target cells, EVs could have many direct clinical applications. The ability of EVs to elicit phenotypic and genotypic changes in cancer presents an opportunity to treat cancer through blocking the transfer of genetic material by preventing EV release 
from cancer cells, or preventing non-malignant cells from accepting the EVs. Additionally, findings from patient-based clinical samples could be implicated in designing therapies not only directed towards the genetic/ epigenetic alterations common to all prostate cancers, but also to those that are unique to each individual patients. Tailoring therapeutic intervention a priori form knowledge of disease course would represent a powerful clinical tool that could lead to improved outcomes and reduced recurrence of prostate cancer.

\section{Additional file}

Additional file 1: Table S1. List of common proteins found in Patient 13,14 , and $16 \mathrm{EVs}$.

\section{Competing interests}

The authors declare that they have no competing interests.

\section{Authors' contributions}

$\mathrm{KP}$ and SC-K contributed equally to this work. KP, SK-C, CD, MD performed all of the experiments. JR provided biopsied patient tissue. LG scored biopsied tumors. KP, SC-K, CD, DP, DM, JR, PQ and DC contributed to the result interpretation and manuscript preparation. DC, JR and PQ conceived the study. KP, DM, PQ and DC participated in its design, coordination and interpretation of the results and finalized the manuscript. All authors read and approved the final manuscript.

\section{Acknowledgements}

This work was supported by the National Institute of General Medical Sciences of the National Institutes of Health under Award Number P20GM103421. The previous segment of this project was supported by the National Center for Research Resources (NCRR) under P20 RR 017695 (DC) and P20GM103468 (PQ). We thank Kate Brilliant for figure and manuscript preparation and Art Salomon for his suggestions and critical review of the manuscript.

\section{Author details}

'COBRE CCRD Proteomics Core, Rhode Island Hospital, Providence, RI, USA. ${ }^{2}$ Division of Hematology/Oncology and Department of Medicine, Rhode Island Hospital, Providence, RI, USA. ${ }^{3}$ Department of Pathology, The Miriam Hospital, Providence, RI, USA. ${ }^{4}$ Department of Urology, The Miriam Hospital, Providence, Rhode Island, USA.

Received: 24 June 2013 Accepted: 16 September 2013

Published: 8 October 2013

\section{References}

1. Han M, Partin AW, Zahurak M, Piantadosi S, Epstein Jl, Walsh PC: Biochemical (prostate specific antigen) recurrence probability following radical prostatectomy for clinically localized prostate cancer. J Urol 2003 169:517-523.

2. Bolla M, Collette $L$, Blank L, Warde $P$, Dubois JB, Mirimanoff RO, Storme G, Bernier J, Kuten A, Sternberg C, et al: Long-term results with immediate androgen suppression and external irradiation in patients with locally advanced prostate cancer (an EORTC study): a phase III randomised trial. Lancet 2002, 360:103-106.

3. Hanks GE, Pajak TF, Porter A, Grignon D, Brereton H, Venkatesan V, Horwitz EM, Lawton C, Rosenthal SA, Sandler HM, et al: Phase III trial of long-term adjuvant androgen deprivation after neoadjuvant hormonal cytoreduction and radiotherapy in locally advanced carcinoma of the prostate: the radiation therapy oncology group protocol 92-02. J Clin Oncol 2003, 21:3972-3978.

4. Pilepich MV, Winter K, Lawton CA, Krisch RE, Wolkov HB, Movsas B, Hug EB, Asbell SO, Grignon D: Androgen suppression adjuvant to definitive radiotherapy in prostate carcinoma-long-term results of phase III RTOG 85-31. Int J Radiat Oncol Biol Phys 2005, 61:1285-1290.

5. Pilepich MV, Winter K, John MJ, Mesic JB, Sause W, Rubin P, Lawton C, Machtay M, Grignon D: Phase III radiation therapy oncology group (RTOG) trial 86-10 of androgen deprivation adjuvant to definitive radiotherapy in locally advanced carcinoma of the prostate. Int J Radiat Oncol Biol Phys 2001, 50:1243-1252.

6. Bavik C, Coleman I, Dean JP, Knudsen B, Plymate S, Nelson PS: The gene expression program of prostate fibroblast senescence modulates neoplastic epithelial cell proliferation through paracrine mechanisms. Cancer Res 2006, 66:794-802.

7. Chang BD, Watanabe K, Broude EV, Fang J, Poole JC, Kalinichenko TV, Roninson IB: Effects of p21Waf1/Cip1/Sdi1 on cellular gene expression: implications for carcinogenesis, senescence, and age-related diseases. Proc Natl Acad Sci USA 2000, 97:4291-4296.

8. Currid CA, O'Connor DP, Chang BD, Gebus C, Harris N, Dawson KA, Dunn MJ, Pennington SR, Roninson IB, Gallagher WM: Proteomic analysis of factors released from p21-overexpressing tumour cells. Proteomics 2006, 6:3739-3753.

9. Krtolica A, Parrinello S, Lockett S, Desprez PY, Campisi J: Senescent fibroblasts promote epithelial cell growth and tumorigenesis: a link between cancer and aging. Proc Natl Acad Sci USA 2001, 98:12072-12077.

10. Shay JW, Roninson IB: Hallmarks of senescence in carcinogenesis and cancer therapy. Oncogene 2004, 23:2919-2933.

11. Keller S, Sanderson MP, Stoeck A, Altevogt P: Exosomes: from biogenesis and secretion to biological function. Immunol Lett 2006, 107:102-108.

12. Abusamra AJ, Zhong Z, Zheng X, Li M, Ichim TE, Chin JL, Min WP: Tumor exosomes expressing Fas ligand mediate CD8+ T-cell apoptosis. Blood Cells Mol Dis 2005, 35:169-173.

13. Muralidharan-Chari V, Clancy JW, Sedgwick A, D'Souza-Schorey C: Microvesicles: mediators of extracellular communication during cancer progression. J Cell Sci 2010, 123:1603-1611.

14. Taylor DD, Gercel-Taylor C: Tumour-derived exosomes and their role in cancer-associated T-cell signalling defects. Br J Cancer 2005, 92:305-311.

15. Valenti R, Huber V, Filipazzi P, Pilla L, Sovena G, Villa A, Corbelli A, Fais S, Parmiani G, Rivoltini L: Human tumor-released microvesicles promote the differentiation of myeloid cells with transforming growth factor-beta -mediated suppressive activity on T lymphocytes. Cancer Res 2006 66:9290-9298

16. Di Vizio D, Kim J, Hager MH, Morello M, Yang W, Lafargue CJ, True LD, Rubin MA, Adam RM, Beroukhim R, et al: Oncosome formation in prostate cancer: association with a region of frequent chromosomal deletion in metastatic disease. Cancer Res 2009, 69:5601-5609.

17. Sandvig K, Llorente A: Proteomic analysis of microvesicles released by the human prostate cancer cell line PC-3. Mol Cell Proteomics 2012, 11:M111. 012914.

18. Chatterjee D, Bai Y, Wang Z, Beach S, Mott S, Roy R, Braastad C, Sun Y, Mukhopadhyay A, Aggarwal BB, et al: RKIP sensitizes prostate and breast cancer cells to drug-induced apoptosis. J Bio/ Chem 2004, 279:17515-17523.

19. Berger R, Febbo PG, Majumder PK, Zhao JJ, Mukherjee S, Signoretti S, Campbell KT, Sellers WR, Roberts TM, Loda M, et al: Androgen-induced differentiation and tumorigenicity of human prostate epithelial cells. Cancer Res 2004, 64:8867-8875.

20. Renzulli JF 2nd, Del Tatto M, Dooner G, Aliotta J, Goldstein L, Dooner M, Colvin G, Chatterjee D, Quesenberry P: Microvesicle induction of prostate specific gene expression in normal human bone marrow cells. $J$ Urol 2010, 184:2165-2171.

21. Zhang $H$, Pelech S: Using protein microarrays to study phosphorylationmediated signal transduction. Semin Cell Dev Biol 2012, 23:872-882.

22. Kasper S, Breitenbuecher F, Heidel F, Hoffarth S, Markova B, Schuler M, Fischer T: Targeting MCL-1 sensitizes FLT3-ITD-positive leukemias to cytotoxic therapies. Blood Cancer J 2012, 2:e60.

23. Mahon KL, Henshall SM, Sutherland RL, Horvath LG: Pathways of chemotherapy resistance in castration-resistant prostate cancer. Endocr Relat Cancer 2011, 18:R103-R123.

24. Covey JM, Jaxel C, Kohn KW, Pommier Y: Protein-linked DNA strand breaks induced in mammalian cells by camptothecin, an inhibitor of topoisomerase I. Cancer Res 1989, 49:5016-5022.

25. Mori S, Chang JT, Andrechek ER, Matsumura N, Baba T, Yao G, Kim JW Gatza M, Murphy S, Nevins JR: Anchorage-independent cell growth signature identifies tumors with metastatic potential. Oncogene 2009, 28:2796-2805 
26. Hermeking H: The 14-3-3 cancer connection. Nat Rev Cancer 2003, 3:931-943.

27. Goldenberg DM, Zagzag D, Heselmeyer-Haddad KM, Berroa Garcia LY, Ried T, Loo M, Chang CH, Gold DV: Horizontal transmission and retention of malignancy, as well as functional human genes, after spontaneous fusion of human glioblastoma and hamster host cells in vivo. Int $J$ Cancer 2012, 131:49-58

28. Gaiffe E, Pretet JL, Launay S, Jacquin E, Saunier M, Hetzel G, Oudet P, Mougin C: Apoptotic HPV positive cancer cells exhibit transforming properties. PLoS One 2012, 7:e36766.

29. Barton BE, Karras JG, Murphy TF, Barton A, Huang HF: Signal transducer and activator of transcription 3 (STAT3) activation in prostate cancer: direct STAT3 inhibition induces apoptosis in prostate cancer lines. $\mathrm{Mol}$ Cancer Ther 2004, 3:11-20.

30. Bromberg J: Stat proteins and oncogenesis. J Clin Invest 2002, 109:1139-1142.

31. Chatterjee D, Moen EL, Chin YE: Inhibition of STAT3 activation by RKIP in colon cancer. Forum on Immunopathological Diseases and Therapeutics 2011, 2:35-45.

32. Fizazi K: The role of Src in prostate cancer. Ann Oncol 2007, 18:1765-1773.

33. Bluyssen HA, Rastmanesh MM, Tilburgs C, Jie K, Wesseling S, Goumans MJ, Boer $P$, Joles JA, Braam B: IFN gamma-dependent SOCS3 expression inhibits IL-6-induced STAT3 phosphorylation and differentially affects IL-6 mediated transcriptional responses in endothelial cells. Am J Physiol Cell Physiol 2010, 299:C354-362.

34. Sen B, Peng S, Woods DM, Wistuba I, Bell D, El-Naggar AK, Lai SY, Johnson FM: STAT5A-mediated SOCS2 expression regulates Jak2 and STAT3 activity following c-Src inhibition in head and neck squamous carcinoma. Clin Cancer Res 2012, 18:127-139.

35. Corbit KC, Trakul N, Eves EM, Diaz B, Marshall M, Rosner MR: Activation of Raf-1 signaling by protein kinase $C$ through a mechanism involving Raf kinase inhibitory protein. J Biol Chem 2003, 278:13061-13068.

36. Chander H, Halpern M, Resnick-Silverman L, Manfredi JJ, Germain D: Skp2B overexpression alters a prohibitin-p53 axis and the transcription of PAPP-A, the protease of insulin-like growth factor binding protein 4. PLoS One 2011, 6:e22456.

37. Wang S, Nath N, Fusaro G, Chellappan S: Rb and prohibitin target distinct regions of E2F1 for repression and respond to different upstream signals. Mol Cell Biol 1999, 19:7447-7460.

38. Fusaro G, Dasqupta P, Rastogi S, Joshi B, Chellappan S: Prohibitin induces the transcriptional activity of $\mathrm{p} 53$ and is exported from the nucleus upon apoptotic signaling. J Biol Chem 2003, 278:47853-47861.

39. Sievers C, Billig G, Gottschalk K, Rudel T: Prohibitins are required for cancer cell proliferation and adhesion. PLoS One 2010, 5:e12735.

40. Radhakrishnan VM, Martinez JD: 14-3-3gamma induces oncogenic transformation by stimulating MAP kinase and PI3K signaling. PLOS One 2010, 5:e11433.

41. Wang Z, Nesland JM, Suo Z, Trope CG, Holm R: The prognostic value of 14-3-3 isoforms in vulvar squamous cell carcinoma cases: $14-3-3$ beta and epsilon are independent prognostic factors for these tumors. PLOS One 2011, 6:e24843.

42. Pan $Y$, Zhong $L$, Zhou $H$, Wang $X$, Chen $K$, Yang HP, Xiaokaiti $Y$, Maimaiti $A$, Jiang L, Li XJ: Roles of vimentin and 14-3-3 zeta/delta in the inhibitory effects of heparin on PC-3M cell proliferation and B16-F10-luc-G5 cells metastasis. Acta Pharmacol Sin 2012, 33:798-808.

43. Murata T, Takayama K, Urano T, Fujimura T, Ashikari D, Obinata D, HorieInoue K, Takahashi S, Ouchi Y, Homma Y, Inoue S: 14-3-3zeta, a novel androgen-responsive gene, is upregulated in prostate cancer and promotes prostate cancer cell proliferation and survival. Clin Cancer Res 2012, 18:5617-5627.

44. Titus MA, Tan JA, Gregory CW, Ford OH, Subramanian RR, Fu H, Wilson EM, Mohler JL, French FS: 14-3-3 $\{$ eta\} amplifies androgen receptor actions in prostate cancer. Clin Cancer Res 2009, 15:7571-7581.

45. Urano T, Takahashi S, Suzuki T, Fujimura T, Fujita M, Kumagai J, HorieInoue K, Sasano H, Kitamura T, Ouchi Y, Inoue S: 14-3-3sigma is downregulated in human prostate cancer. Biochem Biophys Res Commun 2004, 319:795-800.

46. Lodygin D, Diebold J, Hermeking $\mathrm{H}$ : Prostate cancer is characterized by epigenetic silencing of 14-3-3sigma expression. Oncogene 2004, 23:9034-9041.

47. Roy S, McPherson RA, Apolloni A, Yan J, Lane A, Clyde-Smith J, Hancock JF: 14-3-3 facilitates Ras-dependent Raf-1 activation in vitro and in vivo. Mol Cell Biol 1998, 18:3947-3955.
48. Freed E, Symons M, Macdonald SG, McCormick F, Ruggieri R: Binding of 14-3-3 proteins to the protein kinase Raf and effects on its activation. Science 1994, 265:1713-1716.

49. Fischer A, Baljuls A, Reinders J, Nekhoroshkova E, Sibilski C, Metz R, Albert S, Rajalingam K, Hekman M, Rapp UR: Regulation of RAF activity by 14-3-3 proteins: RAF kinases associate functionally with both homo- and heterodimeric forms of 14-3-3 proteins. J Biol Chem 2009, 284:3183-3194.

doi:10.1186/1476-4598-12-118

Cite this article as: Panagopoulos et al.: Reversal of chemosensitivity and induction of cell malignancy of a non-malignant prostate cancer cell line upon extracellular vesicle exposure. Molecular Cancer 2013 12:118.

\section{Submit your next manuscript to BioMed Central and take full advantage of:}

- Convenient online submission

- Thorough peer review

- No space constraints or color figure charges

- Immediate publication on acceptance

- Inclusion in PubMed, CAS, Scopus and Google Scholar

- Research which is freely available for redistribution

Submit your manuscript at www.biomedcentral.com/submit
C) Biomed Central 